\title{
Symbiogenetics
}

\section{COMPARATIVE PHYLOGENETIC ANALYSIS OF SYMBIOTIC GENES OF DIFFERENT NODULE BACTERIA GROUPS USING THE METATREES METHOD}

\section{E.S. KARASEV, E.P. CHIZHEVSKAYA, B.V. SIMAROV, N.A. PROVOROV, E.E. ANDRONOV}

\author{
All-Russian Research Institute for Agricultural Microbiology, Federal Agency of Scientific Organizations, 3, sh. Pod- \\ bel'skogo, St. Petersburg, 196608 Russia, e-mail evgenii1991.karasev@gmail.com (corresponding author) \\ ORCID: \\ Karasev E.S. orcid.org/0000-0003-4240-9587 \\ Chizhevskaya E.P. orcid.org/0000-0002-7715-8696 \\ Provorov N.A. orcid.org/0000-0001-9091-9384 \\ The authors declare no conflict of interests \\ Andronov E.E. orcid.org/0000-0002-5204-262X \\ Acknowledgements: \\ The experiments were carried out using equipment of the ARRIAM Center of Genomic Technologies, Proteomics \\ and Cell Biology. \\ Supported by Russian Science Foundation, project № 14-26-00094П \\ Received December 12, 2016
}

\section{Abstract}

We applied modified phylogenetic analysis method of building meta-trees to study the evolutional patterns of various groups of symbiotic genes (nod-genes control the formation of nodules and nif/fix-genes control symbiotic nitrogen fixation). The method consists in the pairwise comparison of topologies and construction combined dendrograms («meta-trees»), where the relative position of the two trees is a measure of corresponding gene phylogenies congruence. Homologues of 18 symbiotic genes ( $\operatorname{cod} A B C D I J N$, nifABDEHKN, fixABC, $f d x B$ ) are present in each test organism (9 strains belong to the genera Bradyrhizobium, Mesorhizobium, Rhizobium, Sinorhizobium and Neorhizobium). These genes were selected for the implementation of this method, as well as the gene 16S rRNA as a traditional taxonomic chromosome marker. We constructed and compared phylogenetic trees for all these genes and then calculated the pairwise similarity coefficients for their topologies. According to the obtained data we built a meta-tree, and there were two statistically distinct gene clusters identified within. Cluster 1 includes mainly nif- and fix-genes and cluster $2-$ mostly nod-genes, that is related to the data of separate localization of these gene groups in the rhizobial genomes. The exception is the arrangement of nifB and fix $C$ genes with nodA in cluster 2, as well as co-localization of nodI and nifD in cluster 1 . During the identified clusters structure analysis we found strong relationship between the relative gene position and the characteristics of their genome localization in nodule bacteria. Importantly, the differences between clusters 1 and 2 are not expressed less clearly than the differences between nod-and nif/fix-gene groups. It is obvious that clusters 1 and 2 of our meta-tree reflect primarily different mechanisms of nodulation evolution and symbiotic nitrogen fixation associated with the independent origin of the relevant gene groups, and possibly, with their separate horizontal transfer between different groups of rhizobia. Further study of the symbiotic gene evolution in nodule bacteria requires improvements used phylogenetic analysis techniques, including separate analysis meta-tree for rhizobia, representing different stages of symbiosis evolution.

Keywords: phylogenetic analysis, meta-trees, nodule bacteria, symbiotic genes

Soil bacteria of the Rhizobiales order are characterized by the ability to enter into symbiosis with leguminous plants, providing them with products for atmospheric nitrogen fixation, which is accompanied by the formation of nodules on the roots, which provide conditions for effective nitrogen fixation and assimilation of ammonium formed as a result of nitrogenase reaction. However, this group of organisms is of interest not only for microbiology, but also for evolutionary genetics of symbiosis. The analyzed model makes it possible to study the mechanisms of evolution of bacterial genes, which control interactions with different groups of eukaryotes [1]. 
The formation of nodules and symbiotic nitrogen fixation are complex multi-stage processes that are controlled by the genes of both partners. The symbiotic genes of rhizobia are divided into two main groups: virulence genes (nod genes), responsible for signal dialogue with the host plant, as a result of which nodules [2] are formed on its roots, and the genes necessary for symbiotic fixation of atmospheric nitrogen - the nif genes (control the synthesis and regulation of nitrogenase activity) and fix genes (a heterogeneous group of genes involved in the work of the nitrogenase complex, primarily in its supply with electrons and energy) [3].

In most nodule bacteria, nod and nif/fix genes are assembled into groups located in the genome as separate clusters, the structure of which varies in different species. For example, in nodule bacteria of alfalfa (Sinorhizobium meliloti and S. medicae), pea (Rhizobium leguminosarum bv. viciae), clover ( $R$. leguminosarum bv. trifolii), goat's-rue (Neorhizobium galegae) and soybean (Bradyrhizobium japonicum), the main nod genes are closely linked, while the nod $A B C$ genes are organized into one operon (Fig. 1). In many rhizobia, the structural genes of nifHDK nitrogenase are one operon and are closely linked to the nifEN genes. Such organization of nitrogen fixation genes is characteristic for representatives of Sinorhizobium, R. leguminosarum, N. galegae and Mesorhizobium loti. However, in $B$. japonicum, an additional copy of the nifH gene is located outside the nifHDK operon, and the nifA gene is located outside the nif/fix cluster together with the nod genes.

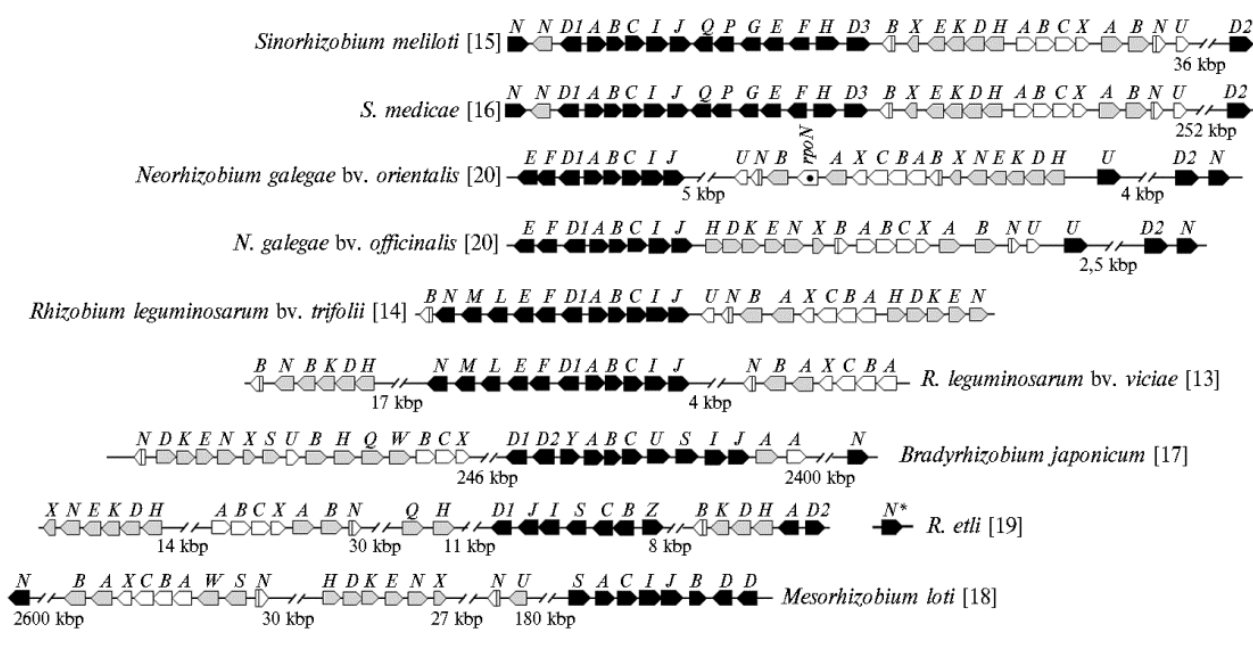

Fig. 1. 1. Location of symbiotic genes in various species of nodule bacteria: - nod genes, $\square-$ nif genes, $\square-$ fix genes, $\mathbb{D}-f d x$ genes, ${ }_{-}-$non-symbiotic genes; the $\operatorname{nod} N$ gene in the genome of $R$. etli (marked with an asterisk) has a chromosomal localization. The schemes are based on the data of genome sequencing of the studied rhizobial strains [4-11].

In evolutionarily advanced species of nodule bacteria (for example, $R$. leguminosarum), symbiotic genes are located on high-molecular plasmids [12]. At the same time, their chromosomal localization is characteristic for the ancestral form - B. japonicum, and also for M. loti [13-14]. There are rhizobia, in which only a few symbiotic genes can be located on the chromosome. This is typical, for example, for $R$. etli: most symbiotic genes are located on the plasmid, and the $\operatorname{nod} N$ gene is located on the chromosome [15] (see Fig. 1).

For a long time, the molecular mechanisms of the evolution of the legumes-rhizobia symbiosis, numbering 60-70 million years [16], remained unclear. Only as a result of the methods of high-performance sequencing, when the rapid accumulation of data on the composition and structure of the genomes of vari- 
ous bacterial species began, the pangenome and metagenomic analysis managed to reconstruct the long path, which micro- and macrosymbionts passed from recruitment into bacterial cells of the nitrogen fixation genes (nif genes) to the development of modern highly specialized legumes-rhizobia symbiotic systems. This process included several stages, in which the groups of genes under consideration were obtained from different sources: nif genes and most fix genes were obtained by ancestral slow-growing rhizobia (Bradyrhizobium) from their freeliving ancestors (Rhodopseudomonas) through vertical inheritance, while the genes for synthesizing lipo-chitooligosaccharide Nod factors responsible for the formation of nodules (nod genes) were obtained from fungi or from gram-positive bacteria during horizontal transfer [17]. The later rapidly growing rhizobia ( $R h i-$ zobium, Sinorhizobium, Neorhizobium) obtained nod and nif/fix genes from ancestral rhizobia during horizontal gene transfer, which led to the formation of special plasmids ( $p$ Sym) containing both groups of sym genes.

The connection of the phylogeny of sym genes with the mechanisms of their origin and evolution, as well as with localization in the rhizobia genomes, has not been adequately studied. For its study, we used a new method of phylogenetic analysis with the construction of "meta-trees" - combined dendrograms, in which the relative position of two trees serves as a measure of the congruence of phylogeny of individual genes. Previously, this method was developed and used to analyze alternative phylogeny obtained in the study of functionally different genes in various groups of eukaryotes (yeasts, fish), in which horizontal gene transfer is limited [18].

In our study, this method was applied to the phylogenetic analysis of two functionally different groups of genes (nod and nif/fix), which are of unequal origin and distributed among taxonomically unrelated groups of rhizobia through both vertical inheritance and horizontal gene transfer. The identified features of the phylogeny topologies constructed for the nodule formation genes and symbiotic nitrogen fixation make it possible to significantly supplement the earlier concept of the directions and mechanisms of the evolution of the gene symbiotic systems in nodule bacteria.

The purpose of the work was to use meta-trees to analyze the contribution of horizontal transfer to the process of evolutionary assembly of the symbiotic gene cluster in rhizobia.

Techniques. Nucleotide sequences of symbiotic genes from the full genomic sequences of 9 representatives of nodule bacteria were used for the analysis: R. leguminosarum bv. viciae 3841 (GenBank GI:115259115) [4], R. leguminosarum bv. trifolii WSM2304 (GI:209537694) [5], S. meliloti 1021 (GI:25168258) [6]，S. medicae WSM419 (GI:150031715) [7], B. japonicum USDA110 (GI:47118316) [8], M. loti MAFF303099 (GI:47118328) [9], R. etli CFN42 (GI:89213252) [10], as well as N. galegae bv. officinalis HA-MBI1141 (GI:659665307) and N. galegae bv. orientalis HAMBI540 (GI:659657635) [11].

Cluster analysis was performed in the computer program MEGA 5.1 (http://www.megasoftware.net/). The alignment of nucleotide sequences was performed using the ClustalW algorithm; the mathematical model when composing the tree for each gene under study was p-distance, the clustering mechanism - Neighbor-Joining. The numerical expression of similarities of phylogenetic tree topologies was calculated using statistical approaches (implemented using the program presented at http://www.mas.ncl.ac.uk/ ntmwn/compare2trees/index.html) [19]. To construct the meta-tree and analyze the reliability of the clustering, the dissimilarity factors were used, which were calculated as the difference of unit (100\%, minuend) and the corresponding similarity factor (subtrahend).

Results. At the initial stage of the work, phylogenetic trees were con- 
structed for each of the following 18 genes: virulence genes $\operatorname{nod} A, \operatorname{nod} B, \operatorname{nod} C$ (responsible for the synthesis of the core part of the Nod factor) [20-22], nodD (in the presence of multiple copies of nodD1, it is a constitutively expressed gene of the flavonoid-sensitive transcriptional activator of nod genes) [23], nodI, nodJ (genes of membrane transporters of the Nod factor) [24], nodN (encodes the dehydratase enzyme) [25]; genes of symbiotic nitrogen fixation fix $A$, fix $B$, fix $C$ (components of the electron transport chain of nitrogenase) [26], nif $A$ (gene of the transcriptional regulator of nif genes), nifB, nifN, nifE (responsible for the synthesis of the Fe-Mo cofactor of nitrogenase), nifD, nifH, nifK (structural components of nitrogenase) [27], $f d x N$ (ferredoxin). The choice was due to the fact that homologs of these genes are present in all representatives of nodule bacteria selected for analysis. In addition, a traditional chromosomal taxonomic marker, the 16S rRNA gene, was included in the study due to the conservation of its nucleotide sequence and low frequency of horizontal transfer.

The methodology for comparing topologies is based on the idea that horizontal transfer of genes from one species of rhizobia to another during evolution introduces significant differences in the topology of the phylogeny of these genes. In the case when the topology of the trees of two genes does not differ, it is assumed that they either do not participate in the horizontal transfer at all, or are transferred together (Fig. 2, A).

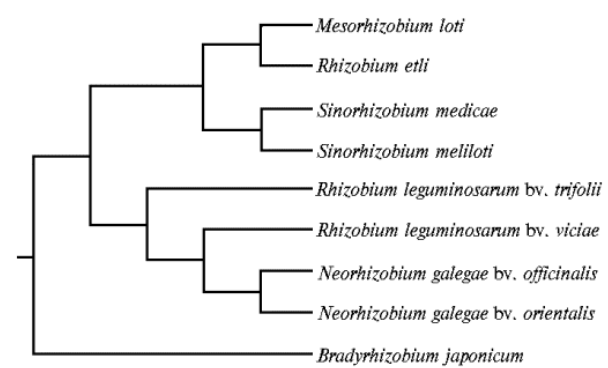

A

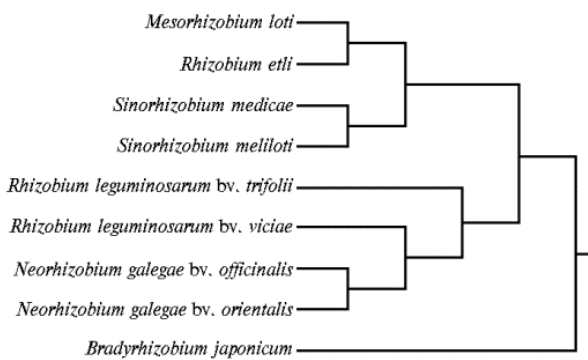

B
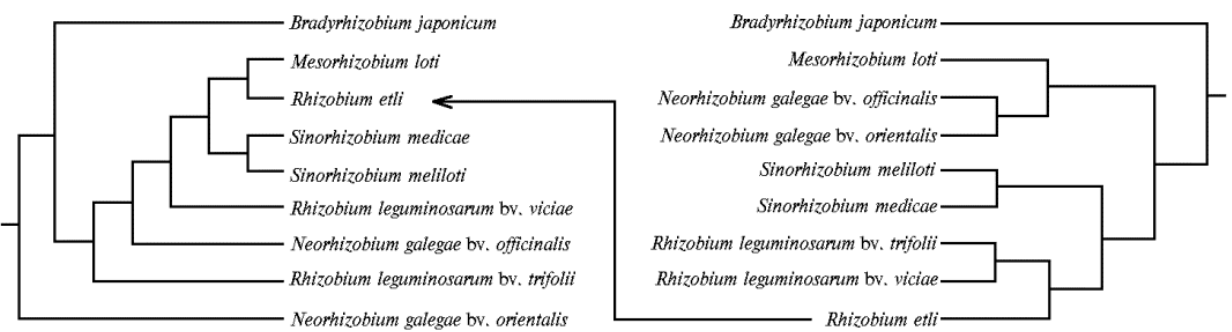

Fig. 2. Comparison of the topology of phylogenetic trees for the nifH (left) and nifN (right) genes with a similarity of $100 \%$ (A) and for the genes $f d x N$ (left) and 16S rRNA (right) with a similarity of $50.5 \%$ (B). The arrow shows the most noticeable difference in topology: the transfer of Rhizobium etli from the $R$. leguminosarum cluster on the 16S rRNA gene tree to the $M$. loti cluster on the $f d x N$ tree.

At the same time, the discrepancy in the tree topology based on the analysis of the sequences of the same set of strains is a result of the horizontal transfer of symbiotic genes (see Fig. 2, B). According to these differences in the topology, we can assume how the horizontal gene transfer occurred during evolution. For example, it can be seen that in the tree for the 16S-rRNA gene in one cluster there are two biovars $R$. leguminosarum and $R$. etli. Since the $16 \mathrm{~S}$ rRNA gene is a chromosomal marker, it can be said that clustered together representatives are evolutionarily similar. At the same time, on the tree of the plas- 
mid marker $f d x N$, rhizobia $R$. etli grouped in the same cluster with $M$. loti, although $R$. etli and M. Loti belong to different families - Rhizobiaceae and Phyllobacteriaceae. This fact allows assuming that there was a horizontal transfer of the $f d x N$ gene between the nodule bacteria $M$. loti and $R$. etli, or these species obtained this gene from a single source during the horizontal transfer.

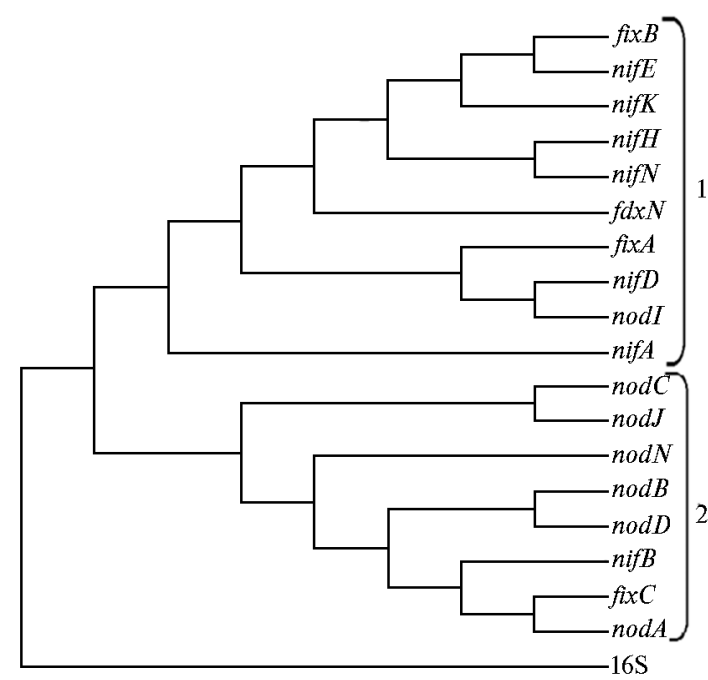

Fig. 3. The main clusters of the meta-tree according to the matrix of similarities of topologies in phylogenetic trees constructed on the basis of the data on the nucleotide polymorphism of symbiotic genes: $1-$ nif/fix cluster, $2-$ nod cluster. concentrated in cluster 1, the group of nod genes - in cluster 2, while the chromosomal marker (16S rRNA gene) did not enter any of the clusters (Table).

Evaluation of the reliability of the meta-tree clustering in accordance with the average distance inside and between clusters/groups of genes using Student's $t$-test

\begin{tabular}{l|c|c|c|c}
\hline \multicolumn{1}{c|}{ Comparison group } & TD & SD & SE & $\begin{array}{l}\text { Reliability of differences ac- } \\
\text { cording to the Student's } t \text {-test } \\
\text { at the significance level p }\end{array}$ \\
\hline Inside the cluster 1 & 0.197 & 0.094 & 0.013 & $<0.001$ \\
Inside the cluster 2 & 0.216 & 0.104 & 0.020 & $<0.01$ \\
Between the clusters & 0.269 & 0.107 & 0.012 & $<0.001$ \\
Inside the nif-fix group & 0.194 & 0.084 & 0.011 & $<0.01$ \\
Inside the nod group & 0.201 & 0.082 & 0.018 & \\
Between the groups of symbiotic genes & 0.270 & 0.099 & 0.011 &
\end{tabular}

N o t e. TD - average difference of topologies, SD - standard deviation, SE - standard error. Comparison of the average distance between tree topologies within one cluster/group, as well as between the trees of one cluster/group and each tree of another cluster/group was performed. The structure of the clusters is shown in Fig. 3.

Such clustering may indicate that in the evolutionary history of the group of genes, nif/fix genes are not related to each other and to chromosomal genes. It is determined by their independent origin and separate horizontal transfer. Indeed, the difference between the groups of nod and nif/fix genes is expressed not less clearly than the dissimilarity between clusters 1 and 2. However, exceptions were found: the nodI gene, which is located in the nif/fix cluster 1 and is transferred along with the nifD gene, as well as the nifB and $f i x C$ genes that enter the nod cluster 2 together with nodA.

As noted above, in the genomes of many nodule bacteria, symbiotic genes are grouped into clusters. In this regard, the joint horizontal transfer of groups of nod and nif/fix genes may be due to the characteristics of their cluster arrangement in the genome (see Fig. 1). However, it is not possible to explain 
the obtained picture only by the specificity of the arrangement of genes on genetic maps. So, in cluster 1 , all the structural genes of nitrogenase were identified, with the genes nifH, nifK, nifE and nifN being grouped together. Such clustering of genes corresponds to their location in the genomes, since in all studied organisms the nifHDK genes constitute one operon, to which the genes nifE and nif $N$ are adjacent. The only exception is the localization of the nif $N$ gene outside the nif/fix zone in nodule bacteria of alfalfa (Sinorhizobium meliloti). Together with the same nod genes, nodN was detected in cluster 2, but it was located separately inside the cluster. It should be noted that the localization of $\operatorname{nod} N$ in the genome may be different. In some representatives, it is located in the same cluster with the nodABC genes ( $S$. meliloti, $S$. medicae, $R$. leguminosarum); in others, it is located separately from the common nod genes. For example, in $R$. ga-legae, the $\operatorname{nod} N$ is located in another nod cluster, and in B. japonicum, M. loti and R. etlioutside the cluster of symbiotic genes. In addition, in $R$. galegae, the $\operatorname{nod} N$ gene has a chromosomal localization.

An interesting result of the analysis was the entry of the nifB and fix $C$ genes into cluster 2 and their grouping together with the nodA gene. It is now known that the genes responsible for the synthesis of the core Nod factor (nod$A B C$ ) are of a non-rhizobia origin. Thus, Hirch et al. [28] analyzed the sequences of bacterial and fungal genes encoding enzymes with catalytic centers similar to those of the nod $A B C$ gene products. It was suggested that the $\operatorname{nod} B$ and $\operatorname{nod} C$ genes could be obtained by rhizobia during the horizontal transfer from gram positive bacteria in which they participate in the biogenesis of the murine cell wall. In addition, these genes could be obtained by nodule bacteria from fungi actively synthesizing chitin-like substances. It is known that many fungi enter into close symbiotic relationships with bacteria, in case of which horizontal gene transfer is quite possible. However, for the $\operatorname{nod} A$ gene, homologous sequences in other organisms were not found. Thus, the $\operatorname{nod} A$ gene has a different origin than $\operatorname{nod} B$ and $\operatorname{nod} C$ [28]. In this regard, its clustering, together with the nif $B$ and fix $C$ genes, can indicate their initially simultaneous horizontal transfer from unidentified organisms.

It is important to note that the specifics of genes clustering on a metatree should be influenced not only by their location in modern forms of rhizobia, but also by evolutionary events that took place before, including independent rearrangements of genomes (see Fig. 1), what is more, both separate genes and genes containing parts of the genome could change their localization. The example of such rearrangements are the inversion of the nif/fix zone in $N$. galegae bv. orientalis [18] and the translocation of nifHDKEN genes in $R$. leguminosarum bv. viciae [11]. There is no doubt that genomic rearrangements have a significant influence on the order of clustering of genes, but the degree of their influence within the framework of the applied model is not clear enough. A significant role in the occurrence of differences in the topologies of the two genes is probably played by the unequal rate of their evolution.

The results obtained in our study do not agree with the data obtained by the methods of molecular phylogeny. Previously, it was shown that the polymorphism of nod genes was formed independently of the divergence of the core elements of the bacterial genome, but correlates with the taxonomy of hosts, whereas the polymorphism of the nif genes correlates with the divergence of the core part of the bacterial genome, but is not associated with the divergence of the host [29]. In our study, the meta-tree method showed the independence of the evolutionary history of both nod and nif/fix genes from the chromosomal marker (16S rRNA gene). Perhaps the method used by us is more sensitive to the influence of evolutionary factors that are not related to the horizontal gene 
transfer and not taken into account in traditional phylogenetic approaches.

Thus, the results of phylogenetic analysis of nod and nif/fix genes, which we carried out using the method of constructing and analyzing meta-trees, confirm the previously identified differences in the evolutionary histories of these genes. The detection of two clusters, one of which consists primarily of nif/fix genes (cluster 1), and the other of nod genes (cluster 2), may indicate the fact that the compared groups of genes originated at different stages in the evolution of rhizobia (in connection with which they occupy different sites on chromosomes or plasmids of rhizobia), as well as the expression of these genes at different stages of symbiosis development (infection of root hairs and release into plant cytoplasm for nod genes, symbiotic fixation of nitrogen - for nif/fix genes). The presence of some nod genes in cluster 1 and some nif/fix genes in cluster 2 may be due to the fact that in the late stages of the evolution of rhizobia, the whole complex of sym genes was transferred as a whole in the composition of Sym plasmids or genomic islands. We cannot exclude the fact that the absence of correlation between the structures of clusters identified in the analysis of the meta-tree and the location of the studied genes on genetic maps is a consequence of intensive intragenomic rearrangements typical for many rhizobia and different rates of evolution of the primary structure in the studied genes.

Further analysis of the role of these evolutionary mechanisms requires a modification of the approach we used, in particular, of the separate analysis of the structures of the meta-trees constructed using samples of strains that occurred at early stages of rhizobia evolution (when horizontal gene transfer was limited due to the chromosomal localization of sym genes in Bradyrhizobium) or at later stages (when horizontal gene transfer was most intense and most likely captured the entire complex of sym genes located on the Rhizobium and Sinorhizobium plasmids). An important purpose of improving the method used is also to develop statistical and bioinformational criteria for supporting clusters that are detected on meta-trees, since in the first stage of the work we used only standard biometric approaches to solve this problem.

Thus, the phylogenetic analysis of nod and nif/fix genes, carried out using the construction and comparison of meta-trees, confirmed the previously identified differences in the evolutionary histories of these genes. The presence of two clusters in the meta-tree, one of which combines mainly nif/fix genes, and the other - nod genes, may be due to the fact that the stages of evolution of the rhizobia, at which the compared groups occurred, did not coincide, and also due to the expression of these genes at different stages of symbiosis development. The absence of correlation between the structure of clusters in the meta-tree and the results of genetic mapping may be due to intra-genomic rearrangements and the unequal rate of evolution of nucleotide sequences of genes. To improve the method used, it is necessary to develop statistical and bioinformational criteria for supporting clusters that are detected on meta-trees.

\section{REFEREN C ES}

1. Provorov N., Tikhonovich I., Andronov E., Belimov A., Borisov A., Vorob'ev N., Dolgikh E., Zhernakov A., Zhukov V., Kimeklis A., Kopat' V., Kurchak O., Onishchuk O., Safronova V., Sulima A., $\mathrm{Chizh}$ evskaya E., Chirak E., S ht a rk O. Geneticheskie osnovy evolyutsii bakterii simbiontov rastenii [Evolution of bacterial symbionts of plants - genetic aspects]. St. Petersburg, 2016 (in Russ.).

2. D o u n i Dzh. V knige: Rhizobiaceae [In: Rhizobiaceae]. St. Petersburg, 2002: 417-434 (in Russ.).

3. Ka mi nski P., B a t u t Zh., B o i st a rd P. V knige: Rhizobiaceae [In: Rhizobiaceae]. St. Petersburg, 2002: 465-492 (in Russ.).

4. Young J., Crossman L., Johnston A. The genome of Rhizobium leguminosarum has 
recognizable core and accessory components. Genome Biol., 2006, 7(4): R34 (doi: 10.1186/gb2006-7-4-r34).

5. R e e ve W., O' H a r a G., Cha i n P. Complete genome sequence of Rhizobium leguminousarum bv. trifolii strain WSM2304, an effective microsymbiont of the South American clover Trifolium polymorphum. Stand Genomic Sci., 2010, 2(1): 66-76 (doi: 10.4056/sigs.44642).

6. B a r net t M., F is he r R., J o n e s T. Nucleotide sequence and predicted functions of the entire Sinorhizobium meliloti pSymA megaplasmid. PNAS USA, 2001, 98(17): 9883-9888 (doi: 10.1073/pnas. 161294798).

7. Reeve W., Chai n P., O'Hara G. Complete genome sequence of the Medicago microsymbiont Ensifer (Sinorhizobium) medicae strain WSM419. Stand Genomic Sci., 2010, 2(1): 77 86 (doi: $10.4056 /$ siqs.43526).

8. Ka neko T., Naka mura Y., S a t o S. Complete genomic sequence of nitrogen-fixing symbiotic bacterium Bradyrhizobium japonicum USDA110. DNA Res., 2002, 9(6): 189-197 (doi: 10.1093/dnares./9.6.189).

9. Ka neko T., Naka mu ra Y., S a t o S. Complete genome structure of the nitrogenfixing symbiotic bacterium Mesorhizobium loti. DNA Res., 2000, 7(6): 331-338 (doi: 10.1093/dnares./7.6.331).

10. G i rard M., Flores M., B rom S. Structural complexity of the symbiotic plasmid of $R h i-$ zobium leguminosarum bv. phaseoli. J. Bacteriol., 1991, 173(8): 2411-2419.

11. Österman J., March J., La i n e P.K. Genome sequencing of two Neorhizobium galegae strains reveals a noeT gene responsible for the unusual acetylation of the nodulation factors. BMC Genomics, 2014, 15: 500 (doi: 10.1186 /1471-2164-15-500).

12. K h a i n es M., F i n a n T. V knige: Rhizobiaceae [In: Rhizobiaceae]. St. Petersburg, 2002: 4162 (in Russ.).

13. Göttfert M., Röthlisberger S., Kündig C. Potential symbiosis-specific genes uncovered by sequencing a 410-kilobase DNA region of the Bradyrhizobium japonicum chromosome. J. Bacteriol., 2001, 183(4): 1405-1412 (doi: 10.1128/JB.183.4.1405-1412.2001).

14. Sullivan J., R o n s o n C. Evolution of rhizobia by acquisition of a 500-kb symbiosis island that integrates into a phe-tRNA gene. PNAS USA, 1998, 95(9): 5145-5149.

15. Go nzález V., Sa nt a maría R., Bustos P. The partitioned Rhizobium etli genome: Genetic and metabolic redundancy in seven interacting replicons. PNAS USA, 2006, 103(10): 3834-3839 (doi: 10.1073/pnas.0508502103).

16. Vorob'ev N., Provorov N. Ekologicheskaya genetika, 2013, 11: 73-85 (in Russ.).

17. Provorov N., Andronov E. Evolution of root nodule bacteria: reconstruction of the speciation processes resulting from genomic rearrangements in a symbiotic system. Microbiology, 2016, 83(2): 131-139 (doi: 10.1134/S0026261716020156).

18. Ny e T. Trees of trees: an approach to comparing multiple alternative phylogenies. Syst. Biol., 2008, 57(5): 785-794 (doi: 10.1080/10635150802424072).

19. N y e T., Li o P., Gilk s W. A nowel algorithm and web-based tool for comparing two alternative phylogenetic trees. Bioinformatics, 2005, 22(1): 117-119.

20. Atkinson E., Pa lci c M., H indsga u 1 O., Long S. Biosynthesis of Rhizobium meliloti lipooligosaccharide Nod-factors: NodA is required for an $\mathrm{N}$-acyltransferase activity. PNAS USA, 1994, 91(18): 8418-8422.

21. John M., Rohrig H., Schmidt J. Rhizobium NodB protein involved in nodulation signal synthesis is chitooligosaccharide deacetylase. PNAS USA, 1993, 90: 625-629.

22. Debelle F., Rose n berg C., De narie J. The Rhizobium, Bradyrhizobium and Azorhizobium NodC proteins are homologous to yeast chitin synthases. Mol. Plant-Microbe Interact., 1992, 5: 443-446.

23. P e ck M., Fis he r R., L o ng S. Diverse flavonoids stimulate NodD $\mathrm{D}_{1}$ binding to nod gene promoters in Sinorhizobium meliloti. J. Bacteriol., 2006, 188(15): 5417-5427 (doi: 10.1128/JB.00376-06).

24. Evans I., D ow n i e J. The NodI product of Rhizobium leguminosarum is closely related to ATP-binding bacterial transport proteins: nucleotide sequence of nodI and nodJ genes. Gene, 1986, 43: 95-101.

25. B a e v N., Shultze M., B a rlie r I. Rhizobium $\operatorname{nod} M$ and $\operatorname{nod} N$ genes are common nod genes: nodM encodes functions for efficiency of nod signal production and bacteroid maturation. J. Bactreriol., 1992, 174(23): 7555-7565.

26. Put noky P., Gross k o p f E., C a m D. Rhizobium fix-genes mediate at least two communication steps in symbiotic nodule development. J. Cell Biol., 1988, 106: 597-607.

27. Arnold W., Rump A., Klip p W. Nucleotide sequence of 24,206-base-pair DNA fragment carrying the entire nitrogen fixation gene cluster of Klebsiella pneumonia. J. Mol. Biol., 1988, 203: 715-738.

28. Hirch A., Lum M., D ow nie J. What makes the rhizobia-legume symbiosis so special? Plant Physiol., 2001, 127: 1484-1492 (doi: 10.1104/pp.010866).

29. Provo rov N., V o r o b'e v N. Geneticheskie osnovy evolyutsii rastitel'no-mikrobnogo simbioza [Genetic aspects of plant-microbe symbiosis evolution]. St. Petersburg, 2012 (in Russ.). 